\title{
Prediction of the wave power in the Black Sea based on wind speed using artificial neural networks
}

\author{
Sorin Ciortan ${ }^{1, *}$, Eugen Rusu ${ }^{1}$ \\ ${ }^{1}$ EMechanical Engineering Department, Engineering Faculty, "Dunarea de Jos" University of Galati, Galati, Domneasca Street, 111, \\ Galati, Romania
}

\begin{abstract}
The paper proposes a prediction methodology for the significant wave height (and implicitly the wave power), based on the artificial neural networks. The proposed approach takes as input data the wind speed values recorded for different time periods. The prediction of significant wave height is useful both for assessment of wave energy as also for marine equipment design and navigation. The data used cover the time interval 1999 to 2007 and it was measured on Gloria drilling unit, which operates in the Romanian nearshore of the Black Sea at about 500 meters depth.
\end{abstract}

\section{Introduction}

It is well known that the wave's height value is important information needed for the activities that intend to recover the energy from waves. The power that a wave can deliver is directly linked [1] to the wave's height. Taking into account that this value is influenced by a lot of factors like wind speed, water temperature, geographic location, date and time, etc., is difficult to predict its evolution [2]. There are out there mathematical models dedicated to this job [3] capable to deliver fairly precise results but there is also room for other ways to do such predictions.

In order to predict wave's height, the main working way is to find all possible influences and render these into mathematical formulas. In the real world, there are so many influences on wave behaviour that even most complex the models can miss some.

Another aspect of wave prediction is linked to the data used for. In order to build a model some previous data must be computed. The model itself will simulate the links between the data and will work only for datasets which have same links between. As anyone can observe, in the late years the wheatear condition are continuously and quickly changing [4]. So, models built on datasets ten, even five, years old can give less precise results for newer ones.

The paper try to prove that a prediction model based on Artificial Neural Networks (ANNs) is suitable for wave prediction if the dataset used are relatively new and more, that for every dataset used for training there is an optimal network architecture.

The ANN based models are neither unique nor new [5] and offer the advantage of "black box" simulation. The influences of all factors taken into account are not transposed into mathematical equations but are implemented "as is" into neural network itself. This way, difficult or even impossible to mathematically simulate interactions can be included.

As target example, the wave height prediction near Gloria (Romanian marine platform) located in the Black Sea, figure 1, was used. In this area, the conditions are appropriate for wave energy acquiring [6] but, also for extreme phenomenon [7].

We try to prove that an ANN based model can be used for significant wave height prediction, but the basic data sets must be related to the domain of weather modifications.

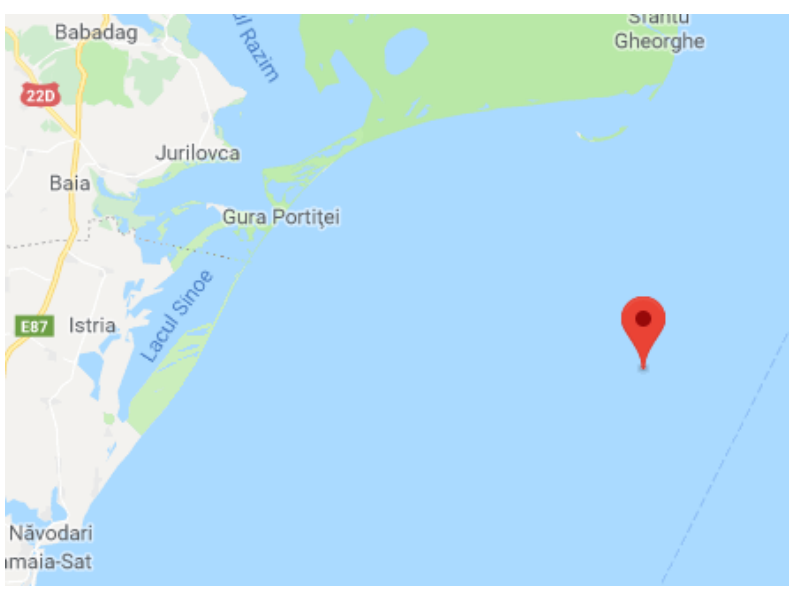

Figure 1. Romanian marine platform location [https://maps.google.com]

\section{Artificial Neural Network based modeling}

Artificial Neural Networks are parallel calculus systems that overcome some disadvantages of sequential calculus machines (base on von Newman architecture) like:

'Corresponding author: sorin.ciortan@ugal.ro 
calculus brake if missing data, compulsory computing algorithm, stop at errors etc.

Basically, an ANN simulate a biological brain, both as architecture and working system. Being composed by many elementary units (so called "artificial neuron") distributed in several areas - layers, [8], figure 2. The ANN can accumulate information, through a "learning or training procedure". During this stage, some known input-output datasets are presented to the ANN and internal links between the neurons are adjusted until the desired results are obtained.

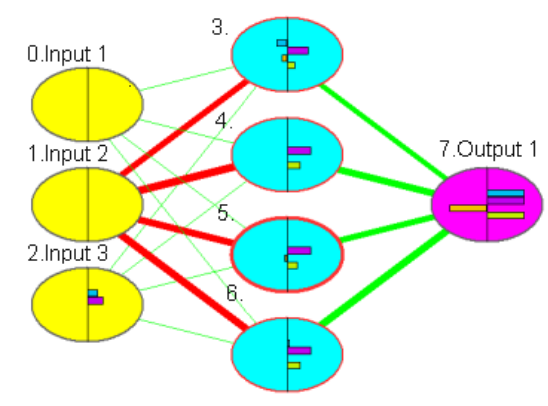

Figure 2. General Feed-forward neural network architecture

After the training procedure is finished, the ANN can be used with several goals: prediction - to find unknown output data for known input data, optimization - to find optimal input values for desired output or analysis - to find which input data has most influence on outputs.

There are several types of ANNs, with different architectures and specific data flows, for this work a feedforward ANN trained with back-propagation algorithm was chosen. Since for every simulated phenomenon the ANN has an optimal architecture (number of neurons and layers), several variants must be tested in order to establish the best one.

ANN based models have also a drawback: the precision is less comparing with a sequential calculus. So, from the beginning, when using a neural model, an error is assumed. Anyway, there are situations when this fact is less important than the other facilities offered.

There are several dedicated software, allowing to create, train and interrogate ANNs in order to obtain predictions. Among these, EasyNN offers all necessary facilities that present work needs.

\subsection{Dataset pre-processing}

In order to be used for ANN modeling, the datasets, must be fitted in appropriate files (tab separated text), one for each year. Due to large number of values (approx. 1450 / year), just one decimal place was kept for wind and wave values. This option facilitates the learning process of the ANN model, taking into account the big differences in wave height over a year $[9,10]$. In figure 3 is presented, as an example, the wave values for 2007.

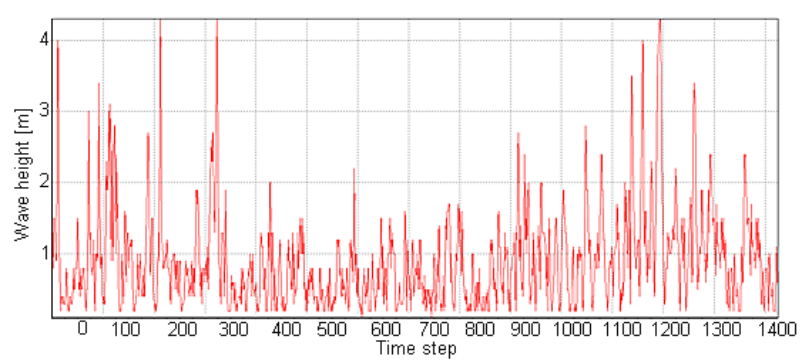

Figure 3. Wave height evolution over year 2007.

\subsection{ANN building and training}

As the establishing of the network architecture represents the most important stage in the ANN model building, an optimization procedure was used: several networks were built and trained for a low number of cycles and the best was chosen. EasyNN has a built-in genetic algorithm based module which performs this task. Taking into account that the wind have the main influence on wave height [11], as training datasets, the values for wind speed and significant wave height were used. In order to cover all the connections between the wind and wave, the datasets contain values at every day/month, for 19992006, with 6 hours time step. The date, time and wind speed stand as inputs and wave height as output. As results, eight ANNs were obtained, one for each year data-set, named further as M-1999....M-2007.

For training procedure, a low learning rate was used, in order to avoid oscillation of the process. As training error the value of 0.1 was targeted. The details are presented in table 1 .

Table 1. The characteristics of the ANN models.

\begin{tabular}{|c|c|c|c|c|c|}
\hline $\begin{array}{c}\text { ANN } \\
\text { code }\end{array}$ & Structure & $\begin{array}{c}\text { Training } \\
\text { cycles }\end{array}$ & $\begin{array}{c}\text { Training } \\
\text { error }\end{array}$ & $\begin{array}{c}\text { Learning } \\
\text { rate }\end{array}$ & Momentum \\
\hline M-1999 & $4-4-4-1$ & 531899 & 0.09 & 0.3 & 0.8 \\
\hline M-2000 & $4-4-4-1$ & 469031 & 0.09 & 0.3 & 0.8 \\
\hline M-2001 & $4-4-4-1$ & 124793 & 0.09 & 0.3 & 0.8 \\
\hline M-2002 & $4-7-5-1$ & 122713 & 0.09 & 0.3 & 0.8 \\
\hline M-2003 & $4-8-4-1$ & 263235 & 0.09 & 0.3 & 0.8 \\
\hline M-2004 & $4-8-4-1$ & 40914 & 0.08 & 0.3 & 0.8 \\
\hline M-2005 & $4-8-4-1$ & 345667 & 0.09 & 0.3 & 0.8 \\
\hline M-2006 & $4-9-3-1$ & 48664 & 0.08 & 0.3 & 0.8 \\
\hline
\end{tabular}

As can be observed in table1, there are differences between the architectures along the years 1999 - 2006. These differences lead to the conclusion that there are some significant changes between the data sets. Usually, an ANN becomes more complex - high number of neurons - when the data are more scattered. This may occur due to climate modification from 1999 to 2006.

In figure 4 it is presented, as an example, the ANN architecture for two different years. 


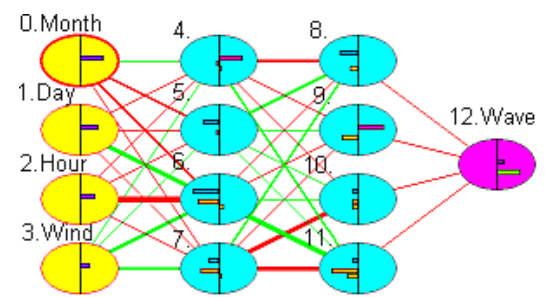

a)

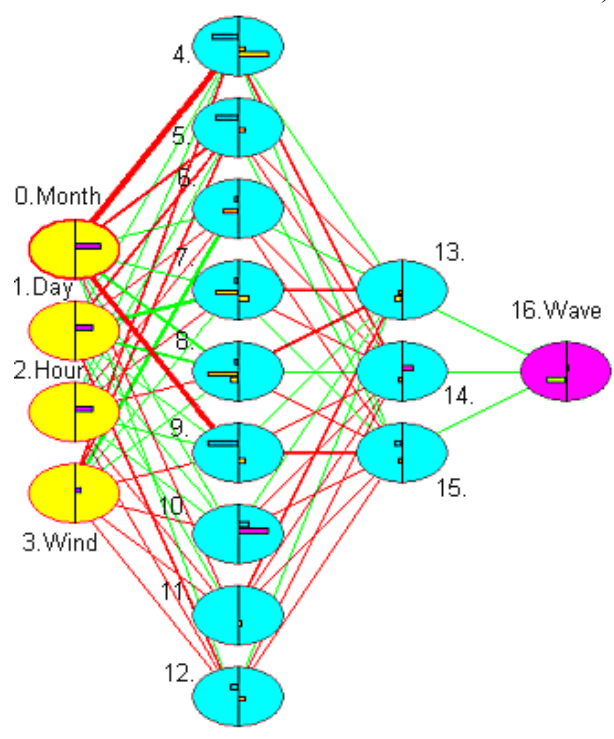

b)

Figure 4. ANN architectures

a) 1999 dataset; b) 2006 dataset

\subsection{ANN model predictions}

With the trained models, the prediction phase can be approached. With this goal, every year dataset was used as input for previous years ANN models and the prediction results were compared with the actual wave height. As significant parameter, the RMSE was used, computed from each time step value for every year. The corresponding results are presented in table 2 .

Table 2. ANN models prediction comparison

\begin{tabular}{|c|c|c|c|c|c|c|c|c|}
\hline & \multicolumn{7}{|c|}{$\begin{array}{c}\text { Prediction precision } \\
\text { (RMSE) }\end{array}$} \\
\cline { 2 - 11 } & 2007 & 2006 & 2005 & 2004 & 2003 & 2002 & 2001 & 2000 \\
\hline M-2006 & 0.190 & --- & --- & --- & --- & --- & --- & --- \\
\hline M-2005 & 0.239 & 0.172 & --- & --- & --- & --- & --- & --- \\
\hline M-2004 & 0.196 & 0.189 & 0.251 & --- & --- & --- & --- & --- \\
\hline M-2003 & 0.171 & 0.190 & 0.214 & 0.174 & --- & --- & --- & --- \\
\hline M-2002 & 0.186 & 0.191 & 0.249 & 0.198 & 0.239 & --- & --- & --- \\
\hline M-2001 & 0.175 & 0.210 & 0.258 & 0.213 & 0.240 & 0.201 & --- & --- \\
\hline M-2000 & 0.163 & 0.211 & 0.248 & 0.181 & 0.265 & 0.199 & 0.198 & --- \\
\hline M-1999 & 0.185 & 0.201 & 0.253 & 0.220 & 0.257 & 0.225 & 0.221 & 0.211 \\
\hline
\end{tabular}

\section{Discussion}

Looking to the results presented in table 2, one can observe that RMSE values are different for different ANN models and, in most cases, these are increasing with the oldness of dataset used for training. This is in well concordance with the hypothesis that during the time the climate conditions are constantly changing. This change modifies the wave height evolution and older models, trained with old datasets gets low in prediction precision. This change is obvious also in graphical representations of wave evolutions, figure 5 .

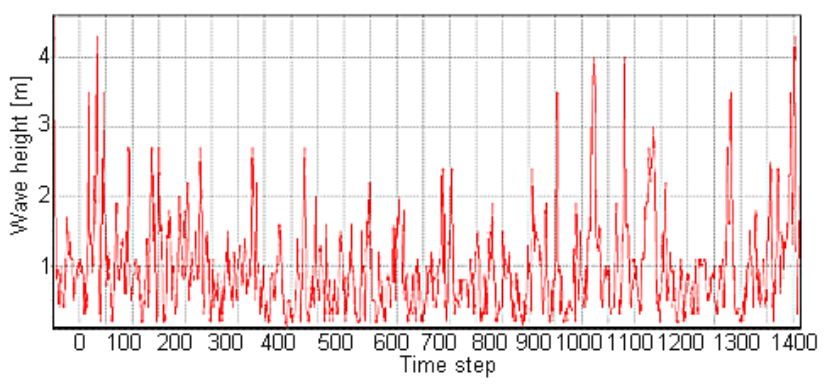

a)

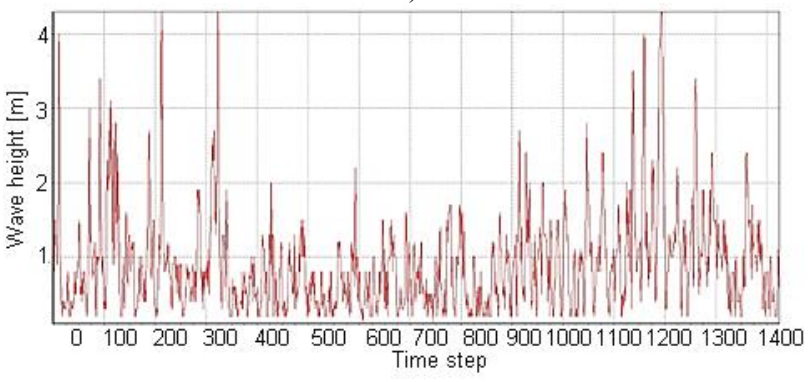

b)

Figure 5. Wave height evolution comparison

a) year 2000 b) year 2007

Another aspect that must be taken into account is the percent of low significant wave height values, comparable to the high ones, present in the training dataset. According to [12], an ANN, during the training stage, will try to emulate the values with greater presence in dataset: low, medium of high. This leads to higher prediction errors when the actual conditions are favourable to other values distribution than data set training. This phenomenon is more obvious in case of low times step values, when height peaks are more likely to be recorded.

For the ANN models here were used datasets covering one whole year, with six hours as value for time step, as a consequence the high wave peaks, due to storms, are present. From this point of view, the obtained RMSE values are consistent with those obtained, in similar conditions, on other datasets $[12,13]$.

\section{Conclusions}

Following all presented above, some conclusions can be drawn:

- The ANN based models can be used successfully for wave height prediction, allowing the wave energy extraction optimization.

- The predictions precision is directly influenced by the datasets used for ANN training, i.e. time step, number of values and high-low values proportion. 
- Another factor of influence on prediction precision is the oldness of training datasets: as these are recent as the prediction is more precise.

- After choosing the appropriate training dataset, the most important step in ANN model building is represented by establishing the architecture. In this purpose a module based on genetic algorithms is highly recommended.

The work is still ongoing and different approaches and datasets are considered.

\section{ACKNOWLEDGEMENTS}

This work was carried out in the framework of the research project REMARC (Renewable Energy extraction in MArine environment and its Coastal impact), supported by the Romanian Executive Agency for Higher Education, Research, Development, and Innovation Funding - UEFISCDI, grant number PN-III-P4IDPCE2016-0017.

\section{References}

1. E. Rusu, Energies 7, 4002-4018 (2014)

2. C. Gasparotti E. Rusu, J. of Env. Prot. and Eco. 13, 1751-1759 (2012)
3. F. Onea, S. Ciortan E. Rusu, En.\& Env. 28, 580597 (2017)

4. L. Rusu,D. Butunoiu, E. Rusu, J. of Env. Prot. and Eco. 15, 445-454 (2014)

5. O. Makarynskyy, D. Makarynska,E. Rusu Mar. Transp. and Exp. of Oc. and Coas. 12,1085 1091 (2005)

6. E. Rusu, J. of Mar. Sc. and Tech. 14, 359-372 (2009)

7. V. Covalenco, S. Ciortan, E. Rusu Mech. Test. \& Diag. 2 16-23 (2017)

8. S. Haykin Neural networks, a comprehensive foundation (MacMillan College Publishing Co., New York, 1994)

9. E. Rusu, Energies 7, 4002-4018 (2014)

10. F. Onea, A. Raileanu, E. Rusu, Advances in Meteorology (2015)

11. F. Onea, E. Rusu, Met. App. 21 316-329 (2014)

12. S.N. Londhe, V. Panchang, J. of Atm. and Oc. tech. 23 1593-1603(2006)

13. B. Paplinska-Swerpel L. Paszke Arch. of HydroEng. and Env. Mech. 53 183-201 (2006) 